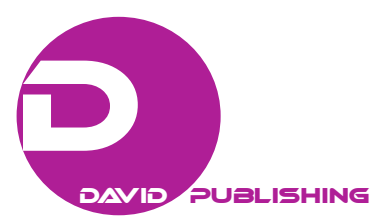

\title{
Econophysical Applications for Economic Progress: Monopoly and Competition Cases
}

\author{
Shahlar Gachay Askerov \\ Baku State University, Baku, Azerbaijan
}

\begin{abstract}
This study attempts to investigate the relationship between monopoly and competition and the philosophy of progress, using the methods, models, and terms from physics. The term "progress" is a newly adopted term and is defined as the value of an increase in the production rate per unit of time. It is shown that to achieve progress, it is necessary to increase the production on a non-linear basis over time. Therefore, it is enough to have many firms that interact with each other under the influence of "market forces". It is important to have a high level of university education, a legal environment for competition and indestructible antitrust laws. Even without strong science, you can make progress by acquiring technology and creating conditions for competition in the market. A factor that strongly influences progress, of course, is technology. For the development of technology, there is a great need for science. Science is a very powerful factor that affects the non-linear change in the economic development. It is shown that during the transition period from an absolute monopoly to an imperfect monopoly the system becomes more complex, and its output characteristics become non-linear as a function of time. It was found that the relationship between monopoly and competition is very simple, and there is no contradiction between them. Initially, the market is born as a monopoly, and then with the creation of similar firms, competition, as the natural market process, begins between them.
\end{abstract}

Keywords: econophysics, progress, competition, monopoly, duopoly, production rate, Schottky diode, revers I-V characteristic Schottky diodes

\section{Introduction}

In the modern world, competition is the driving force of the economy and an important factor in the development of the economy. Therefore, the interaction between monopoly and competition is widely studied. This problem is considered in the light of the econophysics in this article.

Econophysics is an interdisciplinary and standalone research field which has been set to form since the 1990s of the last century. The term "econophysics" was coined by Eugene Stanley (Mantenga \& Stanley, 2000). Nowadays, econophysics is developing rapidly. To solve some economic problems, it is sometimes useful to apply models, methods, and laws of the science of physics. One of the brightest examples for the application of physics to solve economic problems is the gravity model of international trade. This model in 1954 was first introduced in economics world by Walter Isard (1954).

Shahlar Gachay Askerov, doctor of Science, professor, Faculty of Physics, Baku State University, Baku, Azerbaijan.

Correspondence concerning this article should be addressed to Shahlar Gachay Askerov, 23 Zahid Khalilov str, Baku State University, Baku AZ-1148, Azerbaijan. 
In our opinion, some of the results we obtain in different fields of physics can be applied in the market economy. Such phenomenas include low-energy cathode sputtering (Askerov \& Sena, 1969; Askerov, 1970) and the reverse branch of the current-voltage characteristic of the Schottky Diodes (Askerov, Gasanov, \& Abdullayeva, 2018).

\section{Research Methods}

Similarity between the mechanisms of low-energy sputtering and the market economy was show in the report "The Study of the Market Economy Problem by the Method of Econophysics" (Sh.G. Askerov \& A.Sh. Askerov, 2017). To achieve progress in the area of the market economy, one can borrow scientific results obtained in the field of cathode sputtering at low ion energies. Both cathode sputtering and the market economy have a clear relationship between the structure and the property. Thus, it is possible to alter the properties of the object by changing the structure and to convert its linear output characteristics to non-linear characteristic which is very impotent in this case.

Another physical phenomenon that is useful for the market economy is a reverse branch of the current-voltage (I-V) characteristic of the Schottky diodes (SD). Schottky diode is called the diode which is formed between the semiconductor and metal. In this case, semiconductor must definitely have a monocrystalline structure. But the metal may have polycrystalline structure. This type of diode was researched and implemented over the last hundred years. The reverse branches of the current-voltage characteristic is that this diodes vary for their shapes, which are presented in Figure 1. As it is shown in Figure 1, the shapes of the I-V characteristic have "hard" (a) and "soft" (d) breakdowns, and sometimes is observed I-V characteristic with breaks (see Figure 1, b and c).

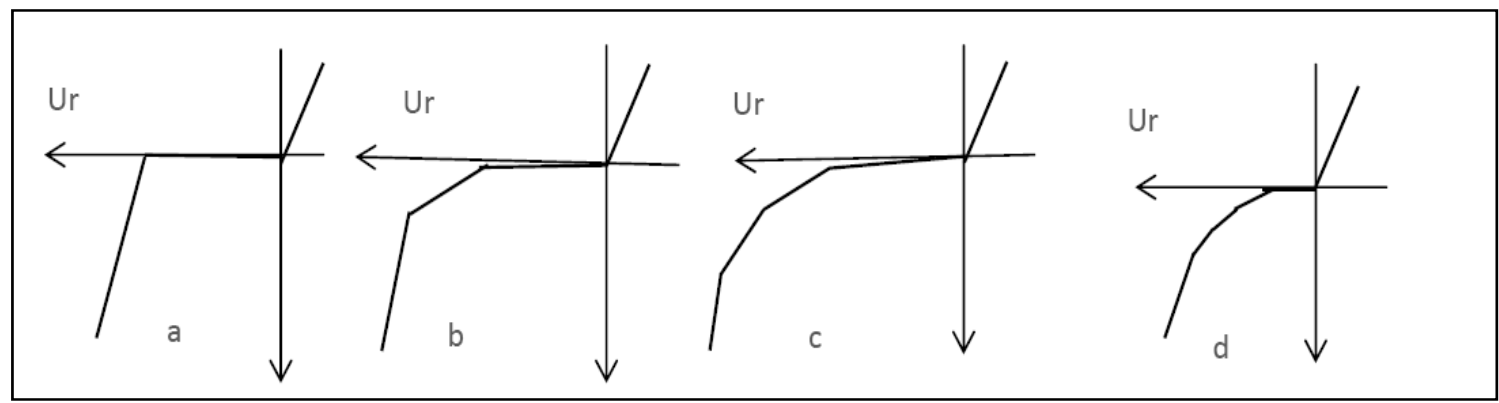

Figure 1. Reverse branches of the I-V characteristic of the metal-semiconductor contact. Note. Ur and Ir show the reverse voltage and the current flowing through the diodes.

Figure 1(a) shows that the SD has an ideal (hard breakdown) I-V characteristic and reverses current breaks only at one point. With an increase in the reverse voltage (Ur), the reverse current grows linearly. This means the interface of investigated diode is uniform and not only semiconductor, but also metal has a monocrystalline structure. And the diode consists only from one sub-diode. Figure 1(b) shows that the reverse I-V characteristic is broken at two points. This means that the investigated diode consists of a parallel combination of two sub-diodes which have different parameters, such as: (i) the breakdown voltage; (ii) diode area; and (iii) barrier heights. After breakdown of the first diode, the reverse current rises according to the avalanche breakdown mechanism. When the voltage reaches to the breakdown voltage of the second diode, the second diode breaks, and the reverse current breaks one more time. In the case, when the number of sub-diodes is three, the third bending appears in the reverse current-voltage characteristic (see Figure 1, c). In the same way, it is possible to 
explain the I-V characteristic of Schottky diodes when the number of sub-diodes are more than 4. Obviously, if the number of sub-diodes is large, then the inverse I-V characteristic of the common diode will be non-linear (see Figure 1, d).

Thus, in the opposite direction, the linearity or non-linearity of the current-voltage characteristic of a SD depends on the degree of homogeneity of the interface. By changing the degree of inhomogeneity with the technology, it is possible to change the number of contact-forming sub-diodes. The number of sub-diodes can be varied by the shape of the I-V characteristic. At $n=1$, the Schottky diode has an ideal "sharp", and at $n>4$, a "soft" current-voltage characteristic. When $n=2$ or 3 , there are İ-V characteristics with 2 or 3 bends. As a result of geometrical addition of linear reverse, currents I-V characteristics, the common current-voltage characteristic becomes soft, i.e., non-linear.

It can be concluded from the aforementioned that, if the numbers of sub-diodes increases, the angular coefficient of the I-V characteristic increases continuously. As a result, the straight-forward character of the sub-diodes is transformed to become non-linear.

According to the author, the above mentioned approach can be applied in the market economy as well.

\section{Results and Discussion}

Let us suppose that there is a linear relationship between the volume of production $(\dot{I})$ and the time $(t)$ :

$$
\dot{I}=b t
$$

Here, $b$ is the proportionality coefficient, which shows the volume of production produced per unit of time. It can also be called the production rate. At $b=0$, there is no production, i.e., commodity is not produced.

We can assume that in the case of a pure monopoly, $b$ remains constant ( $b=b_{m}=$ const) and does not change over time. In this case, we have the production, but we don't have the progress.

To achieve progress, it is necessary that the volume of products produced per unit of time (the speed of production) increases over time. In other words, to achieve progress the linear $\dot{I}(\mathrm{t})$ dependence should become non-linear, or change non-linearly. It means that the in the progress coefficient of proportionality, $b$ (in formula 1) should gradually increase. According to the author, this is a necessary condition for progress.

Figure 2 shows the dependence of the production volume of goods (or services) on time under different market structures. The production activity of the pure monopolist firm is represented by a straight line mm, while the activity of the second similar firm is shown in the straight line dd. As it is easy to see, the presence of the second independent producer of identical goods (dd ) in the market creates competition and the dependence $\dot{I}(\mathrm{t})$ is transformed from a straight line into a broken line 1-2. If another new independent producer of goods (oo lines) appears on the market, the competition will increase even more, the number of broken lines will increase to three (1-2-3).

In the economic theory, the case of 1-2 is called duopoly and the case 1-2-3 is called oligopoly (Econophysics, 2007). The angular coefficient $b$ will respectively increase as the number of firms increases.

The parallelism of the lines mm, dd and oo in Figure 2 means that the technologies used in the production of goods by competing firms are at the same level. In addition, as can be seen from the graph, if the number of competitors grows, the output volume per unit of time will also increase: $b_{o}>b_{d}>b_{m}$.

Thus, in the presence of competition, the transition process from an absolute monopoly to an imperfect monopoly (oligopoly) makes market more complex, and its output characteristics differ significantly from the output characteristics of individual independent producers on the market. 
The increase in the quantity of production per unit of time $b$ in case of duopoly can be represented by the following formula:

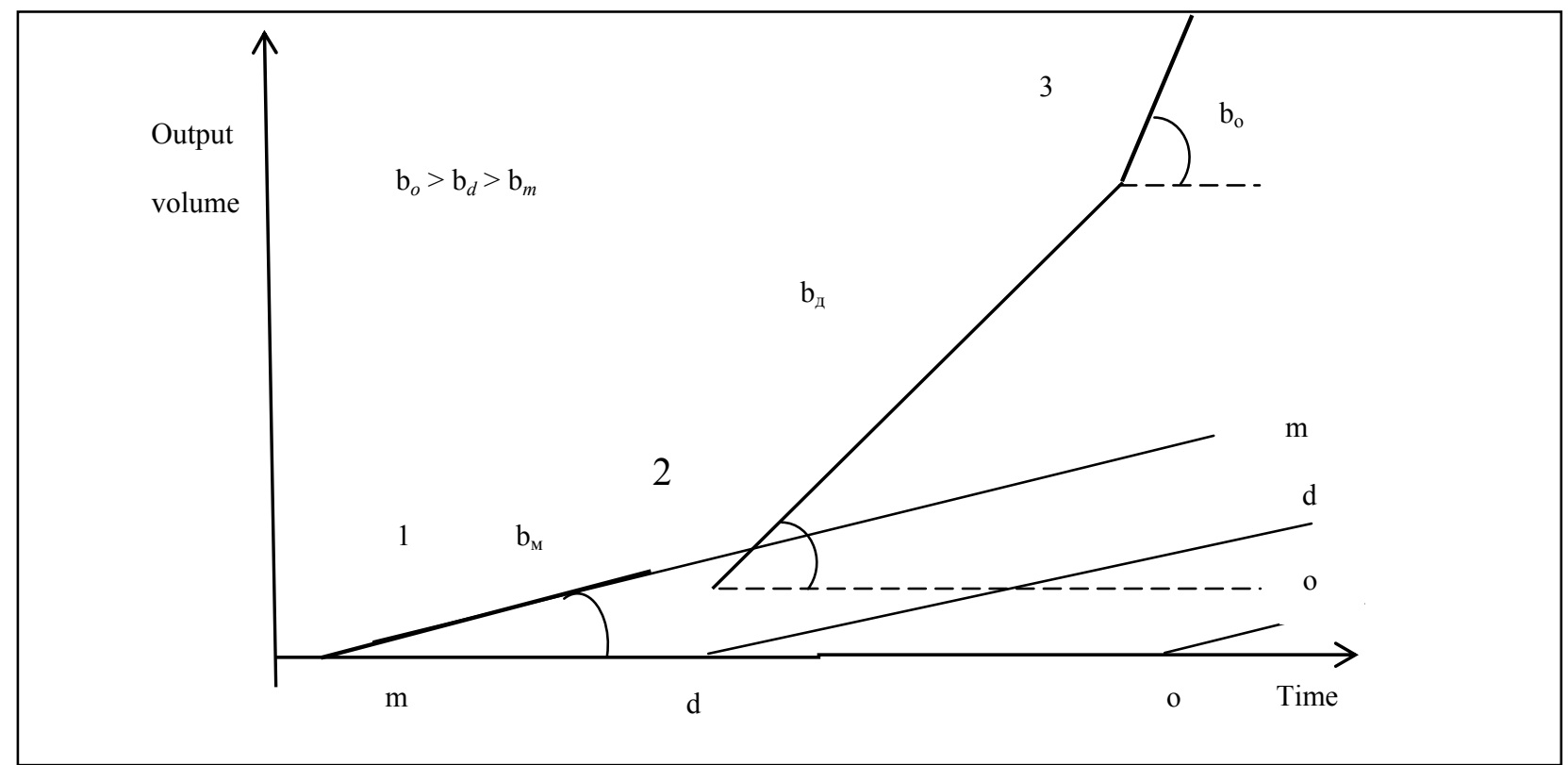

Figure 2. Dependence of the production volume of goods (or service) on time in case of pure monopoly (mm), duopoly (dd) and oligopoly (oo) market structures.

$$
b_{d}=b_{m}+\alpha t
$$

Here, $b_{d}$ and $b_{m}$ are production rates of a duopoly and pure monopolistic firm, respectively; $\alpha$ is a constant characterizing progress. According to the formula (2), progress can be defined as a value equal to the change in $b$ per unit time, and its unit of measurement is the production volume (goods or services) $/ t^{2}$.

It is known from the school physics that, the speed of the alternating motion at any time is defined by the following formula:

$$
v_{t}=v_{0}+a t
$$

where $v_{t}$ is the velocity at time $t$, and $v_{0}$ is the velocity at the time of the reference, and $a$ is the acceleration. Acceleration in physics shows a change in speed over time and measured by the unit: distance $/ t^{2}$. Acceleration in the economy characterizes the progress and shows the change of production rate over time and measured by the unit: production volume $/ t^{2}$.

If we take formula (2) into account in formula (1), we can get that $\dot{I}$ is non-linearly time-dependent. Even with perfect competition (many firms) and an identical technology, the amount of output per unit time proportionally increases over the square of time, which leads to the emergence of progress:

$$
\dot{I}=b_{M} t+\alpha t^{2}
$$

In such conditions, there is no need for the development of science. İt is sufficient to have a high level of university education, and a legal environment for competition. Some countries which belong to so-called emerging economies often choose this way of development.

Another factor that strongly influences the progress is, of course, technology. It is well known that technology depends on the level of science. The need for technological development leads to a greater need for science. For this reason, in developed countries the society allocates big financial resources for science. 
The intellectual way to the progress goes through these stages: science technolog progress.

The relationship between the change in the level of technology $(\Delta T)$ and science $(S)$ in a certain time interval $\Delta T$ can be represented in the following form:

$$
\Delta T=\kappa_{l} S \Delta t
$$

where $k_{1}$ is the coefficient of proportionality.

Figure 3 shows the time dependence of the growth of production of goods by competing firms using different technologies. The graph shows the case where each newly created company uses more advanced technology $\left(b_{o}>b_{\partial}>b_{M}\right)$ for economic growth.

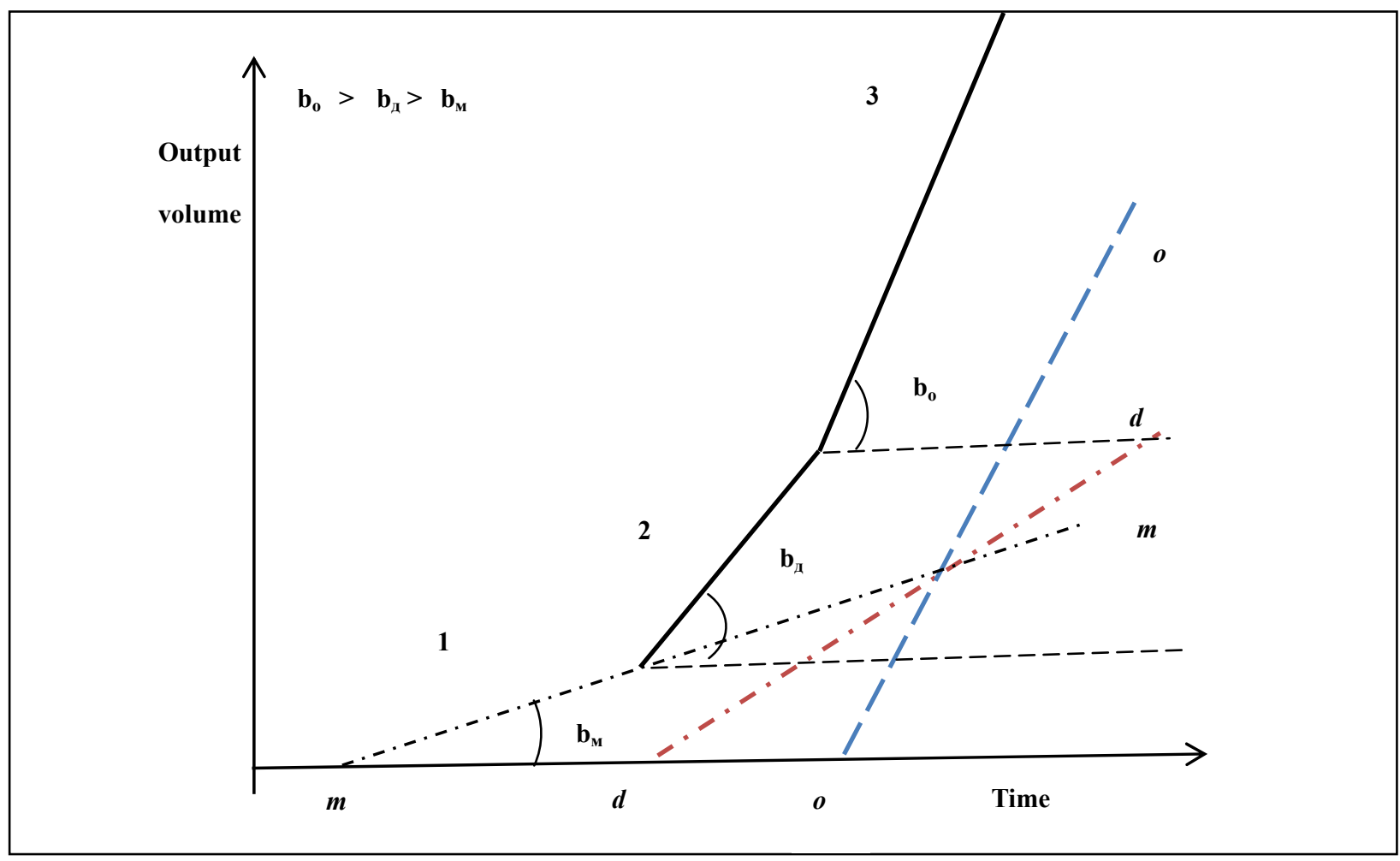

Figure 3. Time dependence of output characteristics of production with application of various technologies: mm-old technology, dd-standard technology, oo-high technology.

As it is seen from Figure 3, technology is a powerful factor to increase productivity over unit a time. For this reason, developed countries are pioneers in education, science, and technology.

Thus, we can conclude that with the increase of the number of competing firms, the types of market structures change, and their sequence from pure monopoly to perfect competition can be represented by the following chain:

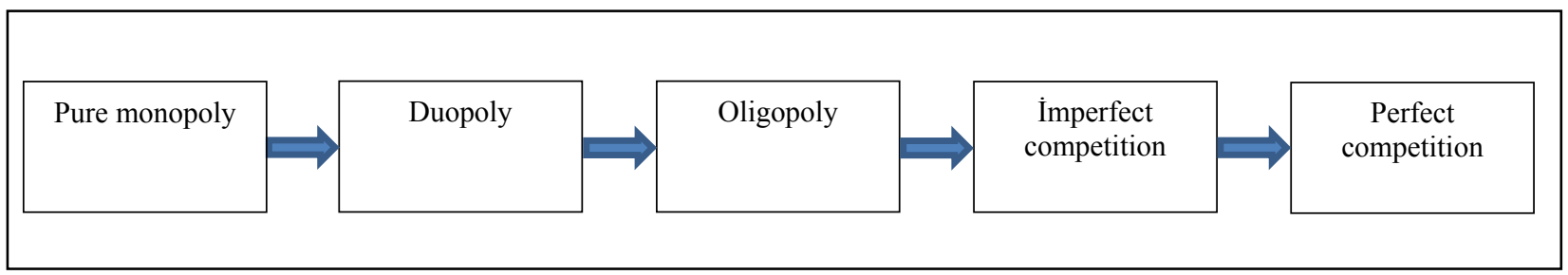

Figure 4. Dynamics of transition from pure monopoly to pure competition. 
As can be seen, the relationship between monopoly and competition is very simple and there is no contradiction between them. Initially, the market is born as a monopoly, and then analogical firms were created, competition between firms starts.

From the foregoing, it can be concluded that in order to achieve progress over time, the production of non-linear products is important. To do this, it is sufficient to have a number of firms interacting with each other under the influence of competition. It is noted that if the number of firms grows, the market is subject to the law of complex systems. By acquiring technology and creating conditions for competition in the market, one can achieve progress even without having a strong science. In other words, free competition and strong antitrust laws are very important for the economic growth of countries.

Technology is a factor that strongly influences the progress. There is a great need for science in order to develop the technology. Science is a very strong factor that influences the non-linearly changing growth of the economy. Scientists are subjects that develop the science. Scientists are generators of ideas; they stand at the center of world development.

Again, as the value of the reverse voltage applied to the Shottky diode increases, the number of sub-diodes involved in the breakdown process increases. As a result, the I-V characteristics of diodes with different shapes are expressed in different linear and non-linear laws. There is also a similar event in the market economy. If the number of firms producing the same product grows, the structure of the market varies, competition takes place, and the quantity of products produced at the unit time increases.

It is shown that with the growth of the number of competing firms, the types of market structures change, and their sequence from pure monopoly to perfect competition can be represented by the following chain: pure monopoly-duopoly-oligopoly-pure competition and again a monopoly arising from technological advancement.

\section{Conclusions}

To summarize, as there is no conflict between monopoly and competition, or there is no "magic hand", there is a natural market interaction. Like an electric charge which creates around itself an electric field, each company creates its own "market field" and interacts with each other through this field. The following results were obtained from this study:

(1) In order to achieve progress, it is necessary to bring together several firms that produce the same product (service). This makes for a complex system and creates a significantly competitive environment. Unlike the straight line characteristics of monopoly firms, the output characteristic of the complex system is non-linearity. The non-linearity of output characteristics is important for progress. Another important prerequisite for progress is the protection of entrepreneurs from monopoly, i.e., and the need for tough anti-monopoly laws.

(2) As a result of the research, the term "progress" has a new meaning, indicating an increase in production rate over a unit time, and measured by the production volume/square of the unit time.

(3) Technology is a key to rapid economic development. It is possible, to acquire technology, to create a competitive environment, and to achieve progress. There is no need for serious science here.

(4) A high technology is essential for speedy development. Technology develops by science, but the science improves by scientists. Hence, scientists are at the center of development. This fact is well-understood by developed countries. Of course, this result is not new, since the values of scientists have been understood by the society since many centuries. 


\section{References}

Askerov, Sh. G. (1970). On the character of the dependence of the coefficient, sputtering on the ion energy in the threshold energy region. Soviet Physics, Solid State, 12, 660-661. (English Translation, Fiz Tverd Tela Trans.)

Askerov, Sh. G. (2018). The study of the market economy problem by the method of econophysics. In 4th International Conference on Global Issues in Multidisciplinary Academic Research, February 01-02, Tokyo, Japan.

Askerov, Sh. G., \& Askerov, A. Sh. (2017). Econophysical analysis of the relationship between monopoly and competition. Journal of Economic Sciences: Theory \& Practice, 74(1), 4-6. Retrieved from http://ecosciences.edu.az/view.php?lang=en\&menu=cjournal\&id=344

Askerov, Sh. G., \& Sena, L. A. (1969). Cathode sputtering of metals by slow mercury ions. Soviet Physics, Solid State, 11, 1288-1293. (English Translation, Fiz Tverd Tela Trans.)

Askerov, Sh. G., Gasanov, M. G, \& Abdullayeva, L. K. (2018). The influence of the metal Microstructure on the breakdown mechanism of schottky diodes. Materials Physics and Chemistry, 1(2), 43-56. doi:10.63019/mpc.v1i2.124

Kharitonov, V. V., \& Ezhova, A. L. (2007). Modern physics in search of economic theory (p. 624). M.: MIPhI.

Kuznetsov, B. G. (2010). Physics and economics: At the roots of econophysics (p. 344). M.: URSS.

Leonidov, A., \& Roumyantsev, E. (2013). Network topology based estimate of systemic risks in the Russian interbank market. Journal of New Economic Association, 3(19), 65-80. (in Russian)

Loskutov, A. Yu., \& Mikhailov, A. S. (2007). Fundamentals of the theory of complex systems. Moscow: Izhevsk.

Mantenga, R. N., \& Stanley, H. E. (2000). An introduction to Econophysics: Correlations and complexity in finance. Cambridge University Press.

Romanovsky, M. Yu., \& Romanovsky, Yu. M. (2012). Introduction to econophysics: Statistical and dynamic models. Moscow: Izhevsk.

Vlasov, M. P., \& Shimko, P. D. (2005). Modeling of economic processes. Rostov-on-Don: Feniks Publication.

Walter, I. (1954). Location theory and trade theory: Short-run analysis. Quarterly Journal of Economics, 68(2), 305.

Zaitsev, S., Zaitsev A., Leonidov, A., \& Trainin, V. (2009). Market mill dependence pattern in the stock market: Multiscale conditional dynamics. Physica A: Statistical Mechanics and its Applications, Elsevier, 388(21), 4624-4634. 